ganzen oder nur im Rahm homogenisiert wird und die so gewonnene, das Fett im homogeniesierten Zustande enthaltende Milch dann nach bekannten Methoden zur Trockne gebracht wird. Durch Wiederanflösen eines solchen Pulvers wird eine Flüssigkeit erhalten, welche durchaus gleiche Eigenschaften wie die Milch hat.

R. Rube \& Co. in Weende bei Göttingen: Verfahren zam Aufbewahren vou Käse mittels Einwickelns in Papier. D.K.P. 184181 vom 5. März 1905. (Patentbl. 1907, 28, 1611.) - Zum Aufbewahren von Käse ist es bekannt, denselben sowohl in sogenanntes Wachsoder Paraffinpapier, d. i. ein durch Tränken mit Wachs oder Paraffin luft- und wasserdicht gemachtes gewöhnliches Papier, als auch in echtes vegetabilisches Pergamentpapier allein einzuwickeln. Bei der Verpackung von Weichkäse aber hat sich weder das eine noch das andere Papier als geniigend widerstandsfähig gegen die Einwirkungen der im Weichkäse enthaltenen scharfen Bestandteile erwiesen. Am besten hat sich noch echtes vegetabilisches. Pergamentpapier bewahrt, wolches aber dennoch in längstens vier Wochen vollständig ver* schimmelt, sich auflöst nnd seinen Zweck nicht mehr erfüilt Um aber den Weichkäse vollständig ausreifen zu lassen, ist es erforderlich, denselben, je nach der Käsesorte, 6-8 Wochen und sogar darüber hinaus eingewickelt zu lassen. Dies wird nach vorliegender Erfindung dadurch ermöglicht, daß man das echte vegetabilische Pergamentpapier mit Paraffin, Ceresin, Stearin, Wachs oder ölhaltigen Stoffen durchtränkt oder überzieht.

A. Oelker.

\title{
Gärungserscheinungen.
}

F. Ehrlich: Die chemischen Vorgänge bei der Hefengärung. (Biochem. Zeitschr. 1906, 2, 52-80.) - Verf. behandelt zunächst kurz die ältere Geschichte der Entwickelung unserer Erkenntnis der Gärungsvorgänge nach der biologischen und chemischen Seite und geht dann auf die Entdeckung der Zymase durch E. Buchner und den Umschwung in den bis dahin gültigen Anschauungen über. Einen weiteren Ausbau erhielt die Buchner'sche Lehre dadurch, daß es nach ähnlichen Methoden gelang, auch aus anderen Hefen und Pilzen zymasehaltige Stoffe auszuziehen, und daß auch bei der intramolekularen Atmung der Pflanze und bei ähnlichen Prozessen im Tierkörper, bei welchen Kohlenhydrate zersetzi werden, zymaseähnliche Enzyme die Hauptrolle spielen. War somit die Bedeutung der Zymase für die alkoholische Gärung des Zuckers außer jeden Zweifel gestellt, so zeigt doch dieses Gärungsenzym in Vergleich zu den bisher bekannten Enzymen so große Unterschiede, daß es sich unter keine Gattung derselben ohne weiteres einreihen ließ, und es entstand namentlich die Frage, wie chemisch im einzelnen durch seine Vermittelung der Abbau des Zuckers zu Alkohol und Kohlensäure zu erklären ist. Buchner und Meisenheimer suchten eine Klärung dieser Frage herbeizuführen, indem sie nach den Zwischenprodukten der Zucker-Alkohol-Gärung forschten. Es zeigte sich, daßB dabei immer inaktive Milchsäure auftritt und daß zugesetzte Milchsäure in manchen Versuchen während der Gärung direkt verschwand. Buchner folgert daraus, då Milchsäure ein normales Zwisehenprodukt darstellt und nimmt an, daß zunächst unter Wasseranlagerung und Abspaltung in anderer Richtung Hydroxylwanderung von den endständigen Kohlenstoffatomen gegen die mittleren erfolge analog der Baeyer'schen Hypothese der Akkumulation des Sauerstoffes und daß dann Sprengung der Kohlenstoffkette da, wo die Anhäufung des Sauerstoffes stattgefunden hat, erfolge. Infolge dieser intramolekularen Oxydation mußte sich zuerst ein Zwischenprodukt, die Dioxy-y-ketonsäure, bilden, das unter Aufnahme eines Moleküles Wasser in zwei Moleküle Milchsäure zerfällt, die ihrerseits wieder durch Wasseranlagerung in je zwei Moleküle Äthylalkohöl und Kohlensäure übergehen. Neuerdings schließen sich Buchner und Meisenheimer der schon von Wolf und Nef geäußerten Ansicht an, daß Methylglyoxal als das erste Umwandlungsprodukt der Glykose bei der Gärung zu betrachten sei. Buchner nimmt außerdem an, daf die totale Vergärung des Zuckers durch zwei Enzyme erfolge, durch die Zymase, die den Zucker in Milchsäure spaltet, und durch die Laktacidase, die Milehsäure in Alkohol und Kohlensäure zerlegt. Auch die Bildung von Essigsäure konnte Buchner für die zellfreie Gärung als charakteristisch erweisen; er vermutet, daß es 
sich auch hier un die Wirkung eines besonderen Enzyms, der Glykacetase, handelt. Das Glycerin und die Bernsteinsäure sind nicht als normale Zerfallprodukte des Zuckers, sondern als Stoffwechselprodukte der Hefe anzusehen. - Verf. behandelt sodann die chemische Zusammensetzung der Hefe, deren genaue Kenntnis erst seit Einführung der Reinzuchtmethoden in den Großbetrieb ermöglicht war. Der Gehalt der Hefe an Enzymen hängt sehr wesentlich von der betreffenden Rasse ab. Bei der Autolyse der Hefe ist die Endotryptase wirksam; sie wirkt nur bei sauerer Reaktion der Flüssigkeit ähnlich wie Pepsin; sie spaltet aber im Gegensatz zu diesem Enzym Eiweißkörper nicht nur bis zu den Peptonen, sondern vollständig bis zu den Aminosäuren, also mit gleichem Erfolg wie das Trypsin, das seinerseits die Spaltung nur bei alkalischer Reaktion vollziehen kann. Der Auf- und Abbau des Hefeneiweißes bietet schließlich auch die Erklärung für die Fntstehung einer Reihe wichtiger Nebenprodukte der Hefengärung im großen, die im wesentlichen aus höheren Alkoholen und Säuren der Fettreihe bestehen, der sogen. Fuselöle. Verf, bespricht die verschiedenen Anschauungen, welche früher über die Entstehung des Fuselöles herrschten und geht dann zum Schluß auf seine eigenen Untersuchungen über, nach welchen die Aminosäuren die Quelle der Fuselöle sind. Es läßt sich aus reinem Leucin, Zucker und Hefe Alkohol mit einem Gehalt bis $\mathrm{zu} 3 \%$ Amylalkohol herstellen. Verf. diskutiert die verschiedenen Möglichkeiten, welche bei der Entstehung der höheren Alkohole aus den Aminosäuren in Betracht gezogen werden können. Er hält es für sehr wahrscheinlich, daß die aus den Aminosäuren entstandenen Oxysäuren in Aldehyd und Ameisensäure gespalten werden und daB auf diese Weise die stete Bildung dieser beiden Gärungsprodukte zu erklären ist. Diese Aldehyde können dann leicht durch Reduktasen der Hefe in den entsprechenden Alkohol und durch Oxydasen in die Säure von gleichem Kohlenstoffgehalt übergehen. Damit wäre auch eine einfache Erklärung des Auftretens von Fettsäuren bei der normalen Gärung gegeben. Außerdem erscheint es möglich, daß infolge einer intramolekularen Oxydation und Reduktion sich aus der Oxysäure direkt der um ein C-Atom ärmere Alkohol bilden kann. Schließlich können alle besprochenen Reaktionen nebeneinander verlaufen. Für die in den Gärungsmaischen und als Spaltungsprodukte der Hefe vielfach beobachteten Aminodicarbonsäuren läßt sich ein ähnlicher Abbau zu Alkoholen annehmen. - Die Reaktion der Fuselölbildung ist aufs engste mit dem Eiweißaufbau der Hefe verknüpft und von diesem unzertrenubar; sie läßt die Entstehung des Fuselöles bei der Vergärung von reinem Zucker und reiner Hefe verstehen. - Die Vergärung des Leucins za Amylalkohol stellt einen neuen sehr eigenartigen Abbau der Aminosäuren dar.

W. Henneberg: EinfluB von zwölf Säurearten, von Alkohol, Formaldehyd und Natronlauge auf infizierte Brennerei- und PreBhefe (Waschen und Reinigungsgärung der Brennerei- und Prefhefe). (Wochensehr. Brauerei $1906,23,527-530,546-549,568-571,580-584$ und 597-602.) Die Veranlassung zu den mitgeteilten Untersuchungen gaben die zahlreichen Anfragen aus der Praxis, wie die Betriebshefen am besten zu reinigen seien, und besonders die Tatsache, dab in unseren heutigen Brennereien und Hefefabriken Bakterieninfektionen noch außerordentlich häufig Störungen hervorzurufen vermögen. In der Hefefabrik sind beiweitem die häufigsten Schädlinge die ,wilden Milchsäurebakterien". Die Preßhefe ist ferner sehr häufig mit Oidium lactis, der Kahmhefe und dem Pinselschimmel, seltener mit Essigbakterien und ganz selten mit Buttersäurepilzen infiziert. Für die Hefefabrik sind die größten Schädlinge die wilden Milchsäurebakterien und die Essigbakterien. Schädlich für die Brennerei sind nur die wilden Milchsäurebakterien. Zum Waschen der infizierten Hefe sind nur diejenigen Stoffe geeignet, die für die Bakterien beträchtlich giftiger sind als für die Hefe. Am meisten geeignet ist Schwefelsäure, dann Salzsäure, Salpetersäure und Phosphorsäure. Mäßig gut geeignet sind Milch- 
säure, Oxalsäure, Weinsäure und Citronensäure. Ungeeignet dagegen sind Flußsäure, Ameisensäure, Essigsäure, Buttersäure, Formaldehyd, Natronlauge und Alkohol. Für die Reinigungsgärung sind geeignet: Schwefelsäure, Salzsäure, Salpetersäure, Milchsäure, Weinsäure und Citronensäure; mäßig geeignet: Phosphorsäure; ungeeignet: Flu@säure, Ameisensäure, Essigsäure, Buttersäure, Oxalsäure, Formaldehyd, Natronlauge und Alkohol. Diese Angaben beziehen sich zunächst nur auf die untersuchten Verhältnisse. An Gift gewöhnte Hefen wurden nicht untersucht.

H. Will.

N. Hjelte Claussen: Vorkommen von Brettanomyces in amerikanischen Lagerbieren. (American Brewers Review 1905, 19, 525.) - Soweit die Erfahrungen reichen, wird mit dem Brettanomyces nur bei obergärigen und vollständig vergorenen Bieren ein reiner und harmonischer Geschmack erreicht. Für jede Art von Hauptgärung ist daher der Pilz völlig unbrauchbar. H. Sebiönning hat aus dänischem Biere den Brettanomyces ebenfalls isoliert. Die Biere muBten jedoch erst einer den Eigenschaften des Pilzes angemessenen Behandlung unterworfen werden, damit sich dieser bedeutend vermehrte. Verf. hat Brettanomyces auch in amerikanischen Lagerbieren gefunden und gehört sein Vorkommen in Lagerbieren überhaupt nicht zu den Seltenheiten. - Da Brettanomyces in sehr versehiedenen Bieren mitunter vorkommt, ist wahrscheinlich eine spärliche, aber einigermaßen andauernde Infelstionsquelle in der Natur vorhanden, von welcher aus eine von der Jahreszeit und lokalen Verhältnissen abhängige, kleine Anzahl von Keimen in die Brauerei eingeführt, aber in der Vermehrung hintangehalten oder unterdrückt wird. (I. Stadium). Findet jedoch der betreffende Organismus ausnahmsweise günstige Bedingungen, so beginnt er unter kräftiger Vermehrung seine spezifische Tätigkeit. Die Verhältnisse in der Brauerei ermöglichen das Entstehen akuter Fälle der eigentlichen Krankheit (II. Stadium). Sind die Verhältnisse in der Brauerei öfters oder gar fortwährend günstig für die Vermehrung, so geht das II. in das III. Stadium über. Der Organismus vermehrt sich massenhaft, und es bilden sich neue Herde, die frisches Infektionsmaterial im kräftigsten Zustande stets verbreiten, unabhängig von allen äußeren Verbältnissen. Die Krankheit ist jetzt chronisch geworden. Der Brettanomyces in den englischen Branereien bietet ein typisches Beispiel einer solchen Infektion im III. Stadium dar.

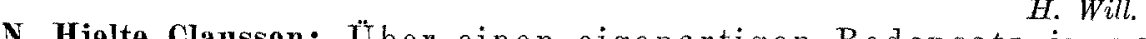
H. Will. steurisiertem Bier (American Brewers Review 1905, 19, 578.) - Verf. erhielt ein pasteurisiertes Bier, welches einen Bodensatz von verhältnismäßig großen, lockeren Flocken enthielt. Diese waren mehr oder weniger dunkelgefärbt und verteilten sich beim Schütteln leicht in der Flüssigkeit, Nach der mikroskopischen Untersuchung bestand der Bodensatz aus einer unorganisierten Masse, in welcher Krystalle, Korkzellen und allerlei unbestimmbare Partikelchen nebst ziemlich zahlreichen Bakterien eingebettet waren. Außerdem wurden noch Diatomeen und grüne Algen in den Flocken gefunden, welche es ermöglichten, bestimmte Folgerungen zu ziehen. Die Brauerei benutzte zum Einweichen der Flaschen eine mittels Seewassers (Lake Michigan) hergestellte Lauge. Die Flaschen wurden nachher mit Seewasser und dann mit filtriertem Wasser gespült. Im Seewasser konnten nun genau die gleichen Arten wie in den Flaschen nachgewiesen werden. Unter den Bakterien zeichnete sich ein Stäbchen aus, das einen sehr voluminösen. Schleim bildete und sich zuweilen so stark entwickelte, daß aufgestellte Wasserproben eine fast ölige Konsistenz annahmen. Wenn eine Flasche mit diesem Wasser ausgespült, daun mit Bier gefüllt und pasteurisiert wurde, bildeten sich ganz ähnliche Flocken, wie sie in den ursprünglich untersuchten Flaschen vorhanden waren. Das gleiche war der Fall, wenn 1 com des Wassers dem Bier in der Flasche vor dem Pasteurisieren direkt zugesetzt wurde. Je mehr Bakterienschleim in den Wasserproben vorbanden war, desto deutlicher kam die Flockenbildung im pasteurisierten Bier zum Vorschein. 
N. Hjelte Claussen: AnläBlich der neuesten Sarcina-Arbeit berichtigende und ergänzende Bemerkungen. (Zeitschr. ges. Brauw. 1906, 23, 339-342.) - Verf. macht einige Bemerkungen zu den Ausführungen von Bettges und Heller (Wochenscbr. Brauerei 1906, 23, 69). Diese beiden Forscher haben die Methode von Claussen zum Nachweis sehr kleiner Mengen von Pediococcus in Hefe für ihre Betriebshefe und ihren Pediococcus vollständig ungeeignet gefunden, indem bei ihren Versuchen nicht die Hefe, sondern der Pediococcus durch die Behandlung mit Fluorammonium getötet wurde. Claussen hat seine Versuche teils mit Carlsberg-Unterhefe I teils mit zwei anderen nahestehenden Reinzuchten von Dortmunder-Hefe ausgeführt. Von 104 Versuchen mit letzterer Hefe baben drej wegen mangelhafter Verteilung der Hefe dasselbe Resultat wie das von Bettges und $\mathrm{Heller}$ erhaltene ergeben. In vielen Fälen sind in der Gelatine vereinzelte Hefekolonien zum Vorschein gekommen, in drei anderen Fällen nur je eine Hefekolonie und in 91 Fällen hat sich gar keine Hefe entwickelt. Die dabei benutzte Arbeitsweise war die folgende: Eine 1/2\% $\%$-ige Lösung des sauren Fluorammoniums mit dem theoretischen Gehalt von ungesättigter Flußsäure wurde jedesmal frisch bereitet. In einem $\mathrm{H}$ an sen'schen Kasten wurden 2-3 g breiiger Hefe und $10 \mathrm{ccm}$ der Lösung in gewöhnliche flambierte Schaugläser gebracht und mit flambierten Glasspateln wenigstens $1 / 2$ Minute möglichst Iräftig gepeitscht. Nach genügendem Peitschen hat die Mischung eine milchweibe Farbe, und die Hefe setzt sich nicht mehr ab. Die negativen Ergebnisse der von Bettges und Heller mit einer Hefe desselben Ursprunges angestellten Versuche sind wahrscheinlich auf ungenügende Verteilung der Hefe oder abnorme Zusammensetzung des angewandten Salzes zurückuführen. Wenn Bettges und Heller mittels der Methode von Claussen den gleichen Pediococcus aus Bier isolieren konnten, so liegt nur die Möglichkeit vor, daß der betreffende Organismus, wenn er wie in der Hefe von Bettges und Heller in der Kokkenform auftritt, weniger widerstandsfähig gegen Flußsäure ist, als wenn er im Bier die typische Pediokokkus-Form annimmt. Möglicherweise waren zwar in den Gelatineplatten Pediokokken in zahlreicher Menge zur Entwickelung gekommen, blieben jedoch infolge der gegenseitigen Beeinträchtigung so klein, daß sie übersehen wurden. Bettges und Heller haben nicht nur die Hefe nicht töten können, sondern es ist ihnen auch nicht geglückt, die Pediokokken in der Gelatine zur Entwickelung zu bringen. Sie sehen hierin ein Zeichen, daß ihr Pediococcus von der Lösung getötet wird und eine Widerlegung der Behauptung von Claussen, daf allen gewöhnlich vorkommenden Bier-Pediokokken dieselbe relative Widerstandsfähigkeit dem sauren Fluorammonium gegenüber beizulegen sei. Bettges und Heller haben jedoch den gleichen Pediococcus mittels der Methode von Claussen isolieren können.

W. Bettges: Zur Sarcinafrage. (Erwiderung auf die berichtigenden und ergänzenden Bemerkungen.) (Wochenschr. Brauerei 1906, 23, 311.) - Da bei der Mitteilung von Claussen der Gedanke aufkommen muB, als sei bei der Nachprüfung der Claussen'schen Methode von seiten Bettges' und Hellers nicht einwandfrei gearbeitet worden, so bringt Bettges eine kurze Berichtigung. Absicht der Mitteilung der beiden Autoren war eine neue bezw. verbesserte Untersuchungsmethode auf Sarcinen zu beschreiben, prinzipielle Fragen dagegen nicht zu lösen. Auf die Arbeitsreise von Claussen wurde deshalb eingegangen, weil die wenig befriedigenden Ergebnisse Anregung zur Ausarbeitung der neuen Methode gegeben hatten. Claussen hat gelegentlich einer Erwiderung an Will und Braun (Zeitschr. ges. Brauerei 1904, 27, 528) die Ergebnisse seiner Untersuchungen als ein fast unantastbares Dogna aufgestellt. Verf. teilt, wie Cla us sen irrtümlich annimmt, den strengen Standpunkt des letzteren in Beziehung auf den alkalisch reagierenden Nähroden nicht. Er hat ausdrücklich bemerkt, daß sein Pediococcus in der 
Nährlösung bei Zusatz von geringen Mengen Ammoniak wächst. Die gleiche Beobachtung ist bei anderen Sarcina-Arten auch von Schönfeld bei Verwendung fester Nährböden gemacht worden. - Bei den Versuchen zum Nachweis einer geringen Infektion von Sarcina mach Cla us s en mit einer halbprozentigen Fluorammoniumlösung wurde genau nach den Angaben, welche Claussen gemacht hatte, vorgegangen. Eine halbe Minute lang gepeitscht, wie neuerdings angegeben, wurde die Mischung allerdings nicht, doch kann man wohl annehmen, daß dieser geringe Untersehied bei der Ausführung der Methode als ausschlaggebend nicht in Betracht kommt. Das zur Trennung angewandte saure Fluorammonium wurde eigens $\mathrm{zu}$ dem $\mathrm{Zwecke}$ bezogen. Die von Claussen für das Misslingen der Versuche von Bettges und Heller vermuteten Ursachen, wie anormale Zusammensetzung des sauren Fluorammoniums, zu stark infizierte Hefe und ungenügende Verteilung derselben lagen demnach nicht vor. Verf. muß daher daran festhalten, dab der in seiner Betriebshefe vorkommende Pediococcus durch eine $1 \%$-ige Lösung von saurem Fluorammonium getötet wurde, wäbrend die von Claussen isolierten Sarcina-Arten eine solche Behandlung aushielten, ohne ihr Leben einzubüßen. Eine 1\%-ige Lösung des Salzes mußte aber benutzt werden, da bei Verwendung einer $1 / 2 \%$-igen Lösung das Wachstum der Hefe nicht unterdrückt wurde, eine Bedingung, welche erfüllt sein mußte, um den Clausse n'schen Nachweis ausführen zu können.

H. Will.

H. Will: Über den Nachweis von Sarcina. (Zeitschr. ges. Brauw. 1906, 29, 577-582.) - Verf, hat die Methode von Bettges und Heller zum Nachweis von Sarcina nachgeprüft und kam dabei zu der Uberzengung, daß sie zu einer allgemeinen Anwendung geeignet ist. Der Nachweis ist nicht nur frühzeitiger, sondern auch sicherer als mit Hefenwasser zu erbringen. Einschlubpräparate sind der Einimpfung in Freudenreich-Kölbchen vorzuziehen. Ein geringer Aciditätsgrad der Näbrlösung schadet der Entwickelung der Sarcina nicht, ebensowenig ein geringerer Grad von Alkalität.

H. Will.

M. Rigaud: Über den Nachweis von Sarcina. (Zeitschr. ges. Brauw. 1906, 29, 599-601.) - Verf. hat anf Veranlassung des Ref. eine größere Anzahl von Jungbieren nach der sogenannten Forcierungsmethode auf Sarcina untersucht. Die hellen Biere waren infolge der stärkeren Hopfung meist frei von Sarcina, die dunklen dagegen fast alle mehr oder weniger infiziert. In 16 Fällen konnte in der Absatzhefe keine Sarcina gefunden werden, in dem forcierten Bier dagegen mühelos. Die Zeit, nach deren Verlauf Sarcina nachzuweisen war, liegt zwischen zwei und drei Wochen. Den Höhepunkt der Entwickelung erreicht die Sarcina in der 4. bis 5 . Woche. Die Forcierung führte also zu brauchbaren Ergebnissen, für den raschen Nachweis genügt sie jedoch nicht. In dieser Beziehung wird sie weit von der Methode, welche Bettges und Heller vorgeschlagen haben, überholt. Immerhin wird sie zur Kontrolle Anwendung finden können.

H. Will.

Die Anwendung getrockneter Agarplatten für den Infektionsnachweis von Luftsarcinen. (Wochenschr. Brauerei 1906, 23, 603-604.) Verf. schlägt zum Nachweis von Sarcina in der Luft Fleischsaftagar vor, welcher in Petrischalen mittels eines Bunsen-Brenners oder einer Spirituslampe getrocknet wird. Die präparierten Schalen stellt man an dem Orte, an welchem die Luft untersucht werden soll, eine Stunde offen auf, schliebt sie nach Verlauf dieser Zeit und bringt sie in den Thermostaten. In zwei oder höchstens drei Tagen entwickeln sich die Sarcina-Kolonien; man sieht gelbe, rote oder rotbraune Punkte von der Größe eines Stecknadelkopfes.

H. Will,

H. Will: Beiträge zur Kenntnis der Sproßpilze ohne Sporenbildung, welche in Brauereibetrieben und deren Umgebung vor- 
kommen. (Zeitschr. ges. Brauw. 1906, 29, 241-243.) - Verf. hat im Jahre 1903 (Zeitschr. ges. Brauw. 1903, 26, 265) über das Verhalten von 15 Sproßpilzen in Würze und Bier sowie gegenüber Bierhefe eine erste Mitteilung gemacht. In der vorliegenden zweiten Mitteilung werden einige Nachträge gebracht, welche sich ebenfalls noch auf Fragen der Praxis des Brauereibetriebes beziehen. Die meisten der Sproßpilze hatten sich bei den früher durchgeführten. Versuchen während der Hauptgärung von untergäriger Bierhefe überhaupt nicht oder nur in sebr geringem Umfang vermehrt. Als Versuchshefen hatten früher die beiden bekannten, extreme Typen repräsentierenden Hefen Stamm 93 und 7 gedient. Später wurde noch eine andere untergärige Bierhefe, Rasse P, dann die bekannten wilden Hefen Saceh, turbidans Hansen und Sacch, validus Hansen geprüft. Von allen Organismen vermochte bei den früheren Versuchen nur der Sproßpilz No. 15 lebhaft gärender Hefe Widerstand zu leisten. Bei dem später mit der Rasse $\mathrm{P}$ durchgeführten Versuch überdauerten auBer No. 15 auch noch die Arten No. 2 und 5 die Hauptgärung. Nach einer Überimpfung in frische Würze war nur No. 2 völlig unterdrückt, No, 5 jedoch schon sehr in den Hintergrund gedrängt. Damit ist ein weiteres Beispiel für die verschiedene Widerstandsfähigkeit der vorliegenden. Sproßpilze gegenüber verschiedenen Rassen von Bierhefe gegeben. Bei der Vergärung der gehopften Würze von 11,5\% B. durch Sacch. turbidans und Sacch. validus blieben nur mehr No. 2 und 15 lebend. Nach einer Überimpfung in frische Würze war auch hier No. 2 völlig unterdrückt. In Bier mit einem Alkoholgehalt von 3,57 Gew. $\%$ und $5,175 \%$ Extrakt vermochten sich mit Ausnahme der Sproßpilzarten No. 7 und 8 alle übrigen mehr oder minder stark zu vermehren. Wenn jedoch das Bier auf der Flasche mit kräftigen Zellen der vorliegenden Organismen infiziert werden sollte, erscheint, wie ein Versuch gezeigt hat, bei gutem, dichtem Verschluß der Flaschen, also bei möglichstem Entzug von Luft, dessen Beeinträchtigung so gut wie ausgeschlossen, um so mehr als durch die Entwicklung der Organismen der Geschmack des Bieres nicht beeinflubt wird.

H. Will.

C. Bergsten: Methode zur Trennung der Mycoderma von den Essigbakterien im Bier durch Anhäufung. (Wochenschr. Brauerei 1906, 23, 596-597). - Verf, teilt eine Methode zur Trennung der Mycoderma von den Essigbakterien im Bier mit, welche er im Beijerinck'schen Laboratorium kennen lernte und weiter ausgearbeitet hat. Hierbei kommen folgende Gesichtspunkte bezw. Eigenschaften der betreffenden Arten in Betracht: 1. Alle Arten von Mycoderma und Essigbakterien sind luftliebend; 2. die Mycoderma bildet keine Säure, die Essigbakterien bilden viel Säure; 3. die Mycoderma kann verhältnismäßig wenig, die Essigbakterien viel Säure vertragen; 4. die Mycoderma ist am besten bei niederer Temperatur entwickelungsfähig, bei höherer Temperatur geht sie allmählich ein; 5. Von den Essigsäurebakterien kann man zwei große Gruppen unterscheiden: a) kryophile, die bei niederer Temperatur am besten wachsen und b) thermophile, die bei höherer Temperatur am besten gedeihen. Durch Versuche wurde festgestellt, wie viel N.-Essigsäure notwendig ist, um die Mycoderma bei den verschiedenen Temperaturen in der Entwickelung zu unterdrücken. - Die Trennung wird in folgender Weise ausgeführt: Man gibt je $100 \mathrm{ccm}$ des zu untersuchenden Bieres in 6 sterile, mit flambierten Uhrgläsern bedeckte Bechergläser von etwa $200 \mathrm{~cm}$ Inhalt oder in FreudenreichKölbchen von gleicher Größe und setzt zu dem bei $15^{\circ} \mathrm{C}$ aufzustellenden Kulturgefäß $25 \%$ sterile N.-Essigsäure, für die Temperatur $20^{\circ} \mathrm{C} 20 \%$, für die Temperatur $25^{\circ} \mathrm{C} 15^{\circ} \%$, für die Temperatur $30^{\circ} \mathrm{C} 10 \%$, für die Temperatur $35^{\circ} \mathrm{C} 5 \%$ und für die Temperatur $40^{\circ}$ keine Essigsäure zu. - Zum Nachweis von Mycoderma läbt man ein wie vorher mit dem zu untersuchenden Biere beschicktes Becherglas oder Freudenreich-Kölbchen, aber obne Zusatz von Essigsäure, im Thermostaten bei $20^{\circ}$ stehen. - Von den Essigbakterien färben sich die meisten Arten mit Jod 
gelb, Bacterium Pasteurianum und Kützingianum blau, ebenso B. xylinum, aber erst nach Zusatz von Schwefelsäure.

H. Will.

E. Buchner und R. Gaunt: Über die Essiggärung. (Liebig’s Anal. 1906, 349, 140.) - Die Verff. haben zur Ausfüllung von Lücken, welche ihre früheren Untersuchungen aufwiesen, neue Versuche mit gewöhnlichen Bieressigbakterien (keine Reinkulturen) angestellt. Zunächst konnte die Wirksamkeit des Dauerpräparates aus Bieressigbakterien in neun verschiedenen Fällen festgestellt werden. Die Menge des vorhandenen Agens schwankte bei sonst gleichen Bedingungen für den Oxydationsvorgang ziemlich stark. Möglicherweise gelingt es nicht immer, die für das oxydierende Enzym schädliche Acetonbehandlung ganz gleichmäBig zu gestalten. Jedenfalls erwies sich die Wirksamkeit auch abhängig von den Züchtungsbedingungen der Organismen. Besonders starke Essigsäurebildung wurde durch die Kultur bei $10-20^{\circ}$ erhalten. Das beobachtete Maximum betrug bei dreitägigem Durchleiten, berechnet auf $100 \mathrm{~g}$ Dauerbakterien, $4 \mathrm{~g}$ Essigsäure. Auf Ton getrocknete Bakterien lieferten wirksamere Präparate, als feuchte. In zwei Fällen haben die Verff. die Bildung von Propionsäure aus Propylalkohol nachgewiesen. Mit diesen Versuchen ist als sicher erwiesen zu erachten, daß die Essigbakterien ihre oxydierende Wirkung der Gegenwart eines Enzyms, einer Oxydase, verdanken, für welche der Name Alkoholoxydase vorgeschlagen wird. Aus den Bakterien hergestellter Preßsaft zeigte bei Luftgegenwart keine oxydierende Wirkung.

H. Will.

W. Henneberg: Zur Kenntnis der Schnellessig- und Weinessigbakterien. (Deutsch. Essigind. 1906, 10, 106-108, 113-116, 121-124, 129 bis 132 u. 137-140.) - Die für die Praxis der Schnellessig- und Weinessigfabriken wichtigen Bakterienarten sind bisher noch nicht genügend bekannt. Verf. beschreibt eingehend 3 neue Arten von Schnellessigbakterien (Bacterium Schützenbachi, B. curvum, B. orleanense), 2 neue Arten von Weinessigbakterien (B. xylinoides, B. vini acetati) und zum Vergleich das B. xylinum. Er bemerkt, daß für die Wissenschaft das strenge Auseinanderhalten der Formen vielleicht ziemlich zwecklos sei, für die Praxis aber nicht. Nach den morphologischen Beschreibungen und den Angaben über das Verhalten gegen verschiedene Temperaturen werden die Wachstumserscheinungen in künstlichen und natürlichen Flüssigkeiten eingehender geschildert. Es folgen dann Beobachtungen über das verschiedene Verhalten gegen verschiedene Essigsäure- und Alkoholmengen und das Verhalten der Kulturen verschiedenen Alters in bezug auf die Entwicklungsfähigkeit in alkoholhaltigen Flüssigkeiten. Die verschiedeneu Arten scheinen verschiedene Ansprüche an das Nährmaterial zu stellen. Bestimmte Regeln lassen sich vorläufig nicht aufstellen. Sie wachsen bei organischem Stickstoff und gleichzeitiger reichlicher Koblenhydratzufuhr am günstigsten. Die Gegenwart von einer bestimmten Menge Alkohol regt die Entwickelung teilweise an. Bei allen geprüften Arten erwiesen sich von den geprüften Nährlösungen als sehr günstig: Bier, ungehopfte Bierwürze mit 3 Vol. $\%$ Alkoholzusatz und verdünnte Getreide. maische mit ebensoviel Alkohol. In Hefenwasser mit Zusatz von Alkohol (ohne und mit $1 \%$ Essigsäure) oder $10 \%$ Robrzueker tritt erst ziemlich spät eine Entwickelung ein, ausgenommen bei B. xylinum und B. xylinoides, bei welchen die Entwickelung bei Rohrzuckerzusatz günstiger erscheint. - Die Essigbakterien sind gegen größere Mengen von freier Essigsäure sehr empfindlich, wenn diese vom Anfang an vorhanden sind. Eine Menge bis zu 1,5\% Säure hat keinen störenden Einflup auf das Wachstum. Allmählich tritt eine Gewöhnung an die Essigsäure ein. Je weniger Alkohol vorhanden ist, desto schneller wachsen die Essigbakterien. Verf. teilt schließlich noch Laboratoriums- und Fabrikversuche mit Reinkultur-Schnellessigbakterien mit. Wenn auch die Arbeit des Essigbildners noch keine ganz normale war, so wurde doch ein absolut klarer und bukettreicher Essig erzielt.

H. Will. 
F. Rothenbach: Zur Systematik der Esaigbakterien. (Deutsche Essigindust. 1906, 10, 193-194.) - Henneberg hat das Verhalten der Essigpilze den Zuckerarten und Stickstoffquellen gegenüber ausführlich behandelt (Deutsche Essigind. 1898, 2, 145). Er hat eine Tabelle aufgestellt, aus der sich die Einwirkung der einzelnen von ihm untersuchten Bakterien auf Zuckerarten und verschiedene andere Körper ergibt. Allerdings führt er in seiner Tabelle nur an, ob eine Säurebildung in der Zucker- u.s.w. -Lösung stattgefunden hat. Ob die Essigpilze aber auf den Zucker oder die anderen Stoffe in denjenigen Fällen, in denen keine Versäuerung beobachtet wurde, anderweitig eingewirkt haben, geht aus der Tabelle nicht hervor. Da die Oxydation von Stoffen häufig mit einer guten Entwickelung von Essigbakterien Hand in Hand geht, während in denjenigen Fällen, in welchen die Essigbakterien überhaupt auf den betreffenden Körper chemisch nicht einwirken, auch meist keine Fntwickelung der Pilze stattfindet, so hat der Verf. die Henneberg'sche Tabelle in der Weise umgeändert, daß an allen denjenigen Stellen, wo Henneberg keine Säurebildung verzeichnet hat, die Entwickelung der betreffenden Essigpilzart - wo eine solche von $\mathrm{Hen}$ neberg beobachtet wurde - kurz vermerkt ist. Bei einer Nachprüfung dee Verhaltens der versehiedenen Essigpilze auf Zuckerarten, Alkohole usw. wird man an der Hand der neuen Tabelle leicht in der Lage sein, an denjenigen Stellen, an welchen keine Säurebillung, aber eine gute Entwickelung der Essigpilze verzeichnet ist, zu ermitteln, ob nicht doch eine Oxydation des betreffenden Körpers, die vielleicht nur bis zur Keton- oder Aldehydbildung reicht, eingetreten ist. $H$. Will.

A. Fischer und M. Kuensberg: Mikroorganismen des Hopfens. (Letters on Brewing 1906, 5, 268-270 u. 366-367.) - Wenn Hopfen, wie bei der Herstellung von Ale, ins $\mathrm{Fab}$ gestopft wird, können die auf ihm vorhandenen Organismen Schaden stiften. Ein eigentümliches Umschlagen des Geschmackes einiger Ales war für die Verff. die Veranlassung die auf Hopfen befindlichen Arten festzustellen. Als Material dienten hierzu verschiedene Sorten Hopfen von Newyork, Kalifornien, Oregon, Saaz und Spalt. Trotz der verschiedenen Gegenden, aus welchen die Hopfen stammten, wurde keine so große Ausbeute an Arten erzielt, als erwartet worden war. Die Höchstzahl von Kolonien (48) entwickelte sich aus einem 1904-er Kalifornia-Hopfen auf Fleischgelatine. Die überhaupt entwickelten Kulturen bestanden aus Hefen, Schimmelpilzen und Bakterien, von letzteren waren Kurzstäbchen vorherrschend. Sarcinen konnten nicht nachgewiesen werden. Von den sieben untersuchten Hopfenmustern wurden neun verschiedene Bakterienarten isoliert. Sämtliche Bakterien wurden in fertiges Flaschenbier geimpft und während 14 Tagen im Thermostaten beobachtet. In fast allen Flaschen trat eine Vermehrung der Bakterien ein. Im Laufe der Zeit waren sie jedoch abgestorben. Schädliche Wirkungen konnten nicht festgestellt werden. Fast alle Kulturen enthielten Torula-Arten, sowohl weiße wie rosa gefärbte. An Hefen wurden von Spalter Hopfen zwei Kolonien isoliert.

\section{H, Will.}

E. Buchner und J. Meisenheimer: Über die Milchsäuregärung. (Liebig's Annalen 1906, 349, 125-139.) - Verf. haben schon früher (Ber. Deutsch. chem. Ges. 1903, 36, 634) drei Versuche mit Dauerpräparaten von Bacillus Delbruecki mitgeteilt, welche eine beträchtliche Milchsäurebildung aus Saccharose durch die mit Aceton getöteten Dauerbakterien bei Toluolzusatz ergaben. Bei zwei weiteren ausführlichen Versuchen mit Dauerpräparaten der gleichen Mikroorganismen lieferten $10 \mathrm{~g}$ der Dauerbakterien aus Saccharose 2,1 bezw. 1,25 g Zinklaktat, was 1,26 bezw. $0,75 \mathrm{~g}$ Milchsäure entspricht. Dauerbakterien, welche eine Stunde auf $90-92^{\circ} \mathrm{er}-$ hitzt, im übrigen aber in gleicher Weise behandelt wurden, bildeten keine Milchsäure. Als Antisepticum wurde Toluol zugesetzt. Bei einem Versuch erbrachten Verff. auBerdem den noch fehlenden strengen Beweis für die Sterilität des Dauerpräparates. 
Durch die vorliegenden Versuche ist einwandfrei erwiesen, daß auch die Milchsäurebakterien, insbesondere Bacillus Delbruecki, die Spaltung des Zuckers zu Milchsäure mit Hilfe eines yon der Lebenstätigkeit der Mikroorganismen abtrennbaren Enzyms bewerkstelligen; welches Verff. als Milchsäurebakterienzymase bezeichnen. Versuche mit Preßsaft, der durch Zerreiben der auf Ton getrockneten Spaltpilze gewonnen wurde, verliefen erfolglos. Dagegen zeigte der Preßrückstand nach Eintragen in Aceton auf Zuckerzusatz gegenüber den direkt aus den frischen Organismen dargestellten Dauerpräparaten unveränderte Gärwirksamkeit bezw. Milchsäurebildung. Das Enzym ist also entweder unlöslich oder es waren bei der Preßsaftdarstellung noch nicht die wirklichen Inhaltssubstanzen der Bakterienzellen in genügendem Maße gewonnen worden. Die Dauerpräparate enthalten auch eine Invertase. Bei der Gärung durch das Dauerpräparat entsteht immer inaktive Säure, gleichgültig, ob man von Saccharose oder vom Milchzucker ausgeht.

H. Will.

C. Wehmer: Über Lebensdauer und Leistungsfähigkeit technischer Milchsäurebakterien. (Chem.-Ztg. 1906, 30, 1033-1035.) Der Verf. berichtet über die Lebensfähigkeit einer nicht näher bezeichneten Milchsäurebakterienart ohne Sporenbildung. Die Art wird seit Jahren in einem 500 cem großen Glaskolben aufbewahrt, in welchem sie mit dem auskrystallisierten, fast steinharten, milchsauren Kalk eingetrocknet ist. Nach 6 Jahren waren die Bakterien noch nahezu ungeschwächt lebensfähig und kaum in ihrer physiologischen Leistungsfähigkeit verändert. Innerhalb der 4 folgenden Jahre fand jedoch ein weitgehendes, wenn nicht völliges Absterben statt. Bei zwei Impfversuchen war das Ergebnis negativ. Eine ähnliche Widerstandsfähigkeit vegetativer Stäbchen gegen das Austrocknen dürfte kaum beobachtet worden sein. Die Widerstandsfähigkeit der Milchsäurebakterien ist eine geringere, wenn die Säure nicht abgestumpft wird. Etwa $100 \mathrm{mg}$ lebender Bakterien setzten den größten Teil des Zuckers (15 g Glykose) in 10,5 $\mathrm{g}$ Milchsäure um, somit ungefähr das Hundertfache ihres Gewichtes, und $1 \mathrm{mg}$ Bakterien erzeugt unter den eingehaltenen Bedingungen pro Tag annähernd $10 \mathrm{mg}$ Milchsäure. Der Verlust an Zucker bei der Milchsäuregärung ist meist ein verbältnismäßig hoher. Der Verf. hat von der vorliegenden Milchsäurebakterienart bei einem ziemlich günstig verlaufenen Versuch aus Glykose 86,14\% Milchsäure erhalten. Die Technil arbeitet noch ungünstiger $(60-70 \%$ Milchsäure auf Glykose berechnet) infolge von Infektionen und unvollkommener Regulierung der Bedingungen.

H. Will.

W. Palladin und S. Kostytschew: Anaerobe Atmung, Alkoholgärung und Acetonbildung bei den Samenpflanzen. (Zeitschr. physiol. Chem. 1906, 48, 214-239.)

T. Takahashi: Eine neue Varietät von Mycoderma als Ursache einer Saké-Krankbeit. (Bull. of the Coll. of Agric. Tokyo 1906, 7, 101-104; Chem. Zentrbl. 1906, II, 621.)

\section{Gebrauchsgegenstände.}

\section{Mineralöle.}

Carl Neuberg: Über die Entstehung optisch aktiver Fettsäuren in der Natur. (Biochem. Zeitschr., 1906, 1, 368-379.) - Die Frage der Entstehung von optisch aktiven Fettsäuren in der Natur hängt eng mit dem Problem der Erdölbildung zusammen, die nach $\mathrm{Engler}$ aus animalischen oder vegetabilischen Fetten erfolgt. Durch die Engler'sche Theorie war aber die optische Aktivität mancher Petroleumsorten nicht erklärt, da Fett und Fettsäuren der heute lebenden Tier- und Pflanzenwelt optisch inaktiv sind. Verf. fand, daß in manchen Fällen das Leichenwachs oder Adipocire optische Aktivität zeigt. Er erklärt dies daraus, daß das Leichenwachs wahrscheinlich ein Abbauprodukt der Proteinstoffe ist, von denen wir dank den Arbeiten E. Fischer's ja wissen, daß neben aromatischen 